\title{
Performance of multi layer feedforward and radial base function neural networks in classification and modelling
}

\author{
M.S. Sánchez ${ }^{a, *}$, H. Swierenga ${ }^{b}$, L.A. Sarabia ${ }^{a}$, E. Derks ${ }^{b}$, L. Buydens ${ }^{b}$ \\ a Department of Mathematics and Computation, Faculty of Science and Food Technology and Chemistry, University of Burgos, Pza. Misael \\ Bañuelos s / n, 09001 Burgos, Spain \\ ${ }^{\mathrm{b}}$ Laboratory for Analytical Chemistry, Faculty of Science, Catholic University of Nijmegen, Toernooiveld 1, 6525 ED Nijmegen, The \\ Netherlands
}

Received 10 February 1995; accepted 6 November 1995

\begin{abstract}
Neural networks have been used in multiple applications, but as a kind of black box for dealing with problems where there is no a priori information about the data. This means that the model is constructed based solely upon information obtained from the data themselves. This seems to be a good property but makes it difficult to validate the models obtained. The classification properties of neural classifiers are usually described by the percentage of correctly classified objects in a test set. Since these straight methods are only based on discrimination, no information can be obtained in a statistical way. In this paper, on a simulated data set, two different types of neural networks, MLF (multi layer feedforward) and RBF (radial base function), are applied to solve a classification problem. The modelling ability, stability and reproducibility of this kind of networks are studied based on various different networks independently trained on the same data set with a predetermined value for the sensibility and specificity. Robustness to different kinds of error is also studied by means of Monte Carlo simulations adding noise at different levels and from different theoretical distributions. Further to this, an analysis based on principal components is carried out to study the apparently different networks obtained. The simulation studies reveal that both types of networks perform well enough to reproduce the input space. For RBF networks, due to the local approach, the study showed some properties related to sensibility and specificity which are relevant in practical problems.
\end{abstract}

Keywords: Multi layer feedforward; Radial base function; Neural networks; Classification; Modelling

\section{Introduction}

Previous work on the predictive ability of radial base function (RBF) and multi layered fcedforward (MLF) neural networks on function approximation problems has been carried out [1] showing the similarities and differences between these two kinds of

\footnotetext{
${ }^{*}$ Corresponding author.
}

networks when they are trained, as far as possible, in the same way and with the same data sets. In the paper mentioned in [1] it is shown that MLF is faster than RBF for fitting problems and, in general, RBF is more robust than MLF for input noise, which means RBF better supports the normal random noise in the input space.

In this paper we focus attention on the differences between MLF and RBF neural networks for classifi- 
cation tasks. Both types of networks are trained with the backpropagation algorithm on the same data set.

In the paper we will maintain a distinction between discriminating and class-modelling techniques [2]. Both fall inside the field of classification but have a clearly different meaning.

The classification rules defined by the discriminating techniques correspond to boundaries that partition the input space into a predefined number of regions, as many as there are classes in the training set. In this way, every object is assigned necessarily to a class. Neural networks can be an example of discriminating techniques.

In class-modelling techniques, the classification rule corresponds to closed boundaries, the input space is divided into as many closed regions ("class boxes") as classes there are, not necessarily disjointed and not necessarily covering the whole input space. This means that, in this case, not every object is assigned to a class, so the classes defined are more specific allowing one to detect objects that are different from the rest ("outliers"); on the other hand, as the classes are not necessarily disjointed, there can be confusion regions, the overlapping regions, where the method is necessarily ambiguous. UNEQ [3] is a typical example of a class modelling technique.

From the statistical point of view, a model can be considered as a hypothesis test [4] for each class in the problem: the null hypothesis, $\mathrm{H}_{0}$, is that a specific object belongs to a class and the alternative hypothesis, $\mathrm{H}_{\mathrm{a}}$, is that this object does not belong to this class. In this case, one can select a value for the probability of rejecting $\mathrm{H}_{0}$ if it is true, $\alpha$, and looks for a strategy to define the critical distance such that the probability of accepting $\mathrm{H}_{0}$ if it is false, $\beta$, be minimum. In other words, $\alpha$ is the significance level of the test, i.e. the probability of false positive (probability of error type I), whereas $\beta$ is the probability of false negative (probability of error type II). In this way the set of objects whose distance to the model is greater than the critical distance, defines the (mathematical) complement of the class box. Thus, the probability $\beta$ is a measure of the quality of the model.

But it is not always possible to compute exactly the values for $\alpha$ and $\beta$, so their estimations have to be used. In this sense, an estimation of the $1-\alpha$ value is the sensibility of the model, that is, percentage of objects belonging to a class that are correctly classified into this class, while $1-\beta$ can be estimated as the specificity of the model, percentage of objects not belonging to a class that are correctly classified as being outside the class.

Both the specificity and the sensibility are related. The sensibility can be increased using a higher value for the critical distance (thus "increasing" the class boundaries); more objects are within the class and the sensibility will increase. But when the boundary of a certain class is higher, the specificity of the model will decrease.

For classification problems neural networks are used, in general, in well-defined problems, for example, to evaluate if an object is acceptable or not in a multivariate quality control process. In this case, one is dealing with a discriminant problem and the training of the network is carried out by evaluating the performance of the network as discrimination method, i.e. by means of the percentage of correct classifications. But, when the network is used to predict whether a new object is acceptable or not, it should be taken into account that variations in the production process can provide characteristics to the new object different from those which the objects have in the training set. In this case, the problem is a modelling problem: the question now is to detect that the object is different. So, a problem which is posed and solved as a discriminating problem becomes a modelling problem. It is interesting to know the ability of the networks to react to this situation.

It is in this context that the results shown in this paper should be evaluated. The networks are trained to solve a discriminant problem, and then attempts to make a model with the outputs of the networks are done based on different criteria to establish the class boundaries [2]. Thus, much attention is paid to the additional information one can obtain with a modelling technique: the sensibility and specificity computed based on various criteria to define a model with the outputs obtained with both type of networks.

\section{Theory}

Both MLF and RBF neural networks are known from the literature [5-7] as well as scveral applications $[8-10]$. Here we will only write the basic for- 
mulae in order to describe the networks uscd and to uniform the notation.

For both kind of networks, there are theoretical results (see for instance [11] for RBF and [12] for MLF) which show that only one hidden layer is enough to make the networks universal approximators.

Based on the mentioned theoretical results and, as far as possible, the networks were configured similarly: both types are three layer fully connected neural networks, this means, only one hidden layer was used with a variable number of hidden units and a linear output layer.

In both cases the networks receive information from the "real world" and pass it to the hidden layer by means of a distributional layer, the input layer of the network. In the hidden layer, the information is processed and the resulting signal is sent to the output layer that acts as a linear regression between the outputs of the hidden layer and the target output, the response we try to obtain.

The main difference between MLF and RBF networks is the way their hidden layer processes the information it receives:

- An MLF network uses logistic functions in its hidden unit. The most usual, and that used in this paper, is the sigmoidal function defined as:

$\psi(x)=\frac{1}{1+\exp [-(x+\sigma)]}$

where $\sigma$ is the bias term used to shift the sigmoid function along the abscissa axis.

Each unit in an MLF-hidden layer computes an hyperplane in the input space. These kinds of transfer functions act as soft-limiter functions using the computed hyperplanes as discriminant hyperplanes to separate the classes in the training set.

- An RBF network works as a kind of kernel estimation method. It approximates an unknown function by the weighted sum of different kernel functions, the radial basis functions, which are used in the hidden layer of an RBF network. The most common kernel function is the Gaussian function defined as:

$$
\psi(x)=\exp \left(\frac{-x^{2}}{\sigma^{2}}\right)=\frac{1}{\exp \left(\frac{x^{2}}{\sigma^{2}}\right)}
$$

where $\sigma$ is a specific parameter of the radial function (the width) that represents the "portion" of the space under the influence of the kernel function. So, the RBF networks divide the input space into regions (not necessarily disjointed) each under the influence of one (or more) radial function, like a clustering technique.

For classification problems, a simple modification of the Gaussian function can be used [13] to improve the performance of an RBF network. The modified kernel function is defined as:

$$
\psi(x)=\frac{1+R}{R+\exp \left(\frac{x^{2}}{\sigma^{2}}\right)}
$$

where the $R$ parameter was added to the original Gaussian function. The performance of an RBF network for classification tasks is enhanced by the addition of the factor $R$ to the Gaussian kernel function. The optimal values for $R$ are problem dependant and should be fixed keeping in mind the relationship between $R$ and $\sigma$. The $\sigma$ parameter still determines the portion of the space under the influence of the kernel function (see Fig. 1a). The $R$ parameter flattens the kernel function around zero (Fig. 1b) so the outputs of the objects lying within the influence region of the function are similar and near one (the highest value the function can allow). For function approximation, RBF networks have a better performance with low values for $R$ and $\sigma$, whereas for classification tasks bigger values give better results. This is because RBF networks model a kernel function around a certain cluster of points in the input space. Within these clusters the responses of the kernel function should be more or less the same while at the boundaries of cluster the responses of the kernel function should change quickly to zero. With this modification and an appropriate value for $R$, objects belong to the same class, supposing they are close to cach other (thus in the same influence region), obtain the same value as output from the hidden layer. So, the network needs fewer hidden units and it is easier for the final linear regression to separate the classes.

Once the topology for both types of networks is decided and the different weights are initialized, the output of the network can be obtained. In this step, some criteria must be defined to say whether the re- 

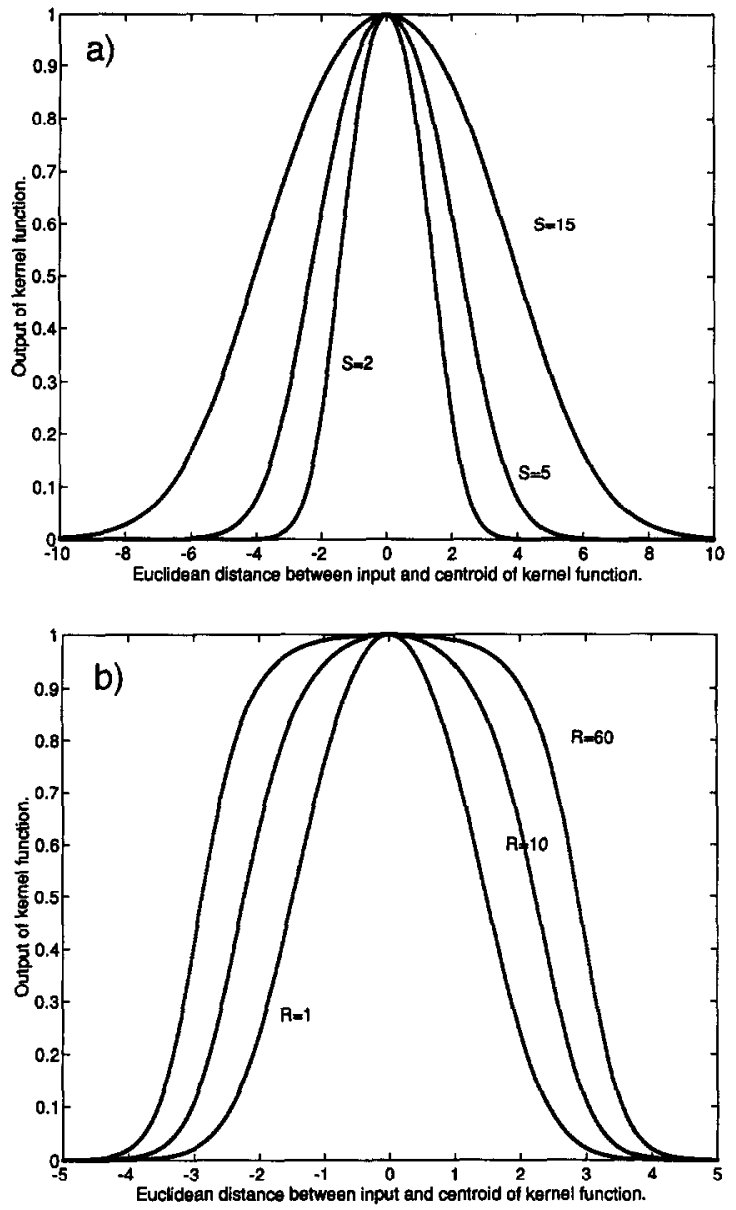

Fig. 1. Modifications of the Gaussian function. (a) Effect of the $S$ parameter (squared width of the kernel function). $R$ was constantly equal to 1 . (b) Effect of the $R$ parameter in flatting the Gaussian kernel function. $S$ was fixed at 2 .

sponse is acceptable or not. If it is not, there are some learning algorithms, both supervised and unsupervised, to train the network until the acceptable response is reached.

Above all for RBF networks several different training algorithms are proposed to find the optimal settings of the networks. One of the methods is the training by gradient descent through backpropagation [14].

This was the method used to train both types of networks, although this is a learning algorithm specially suited for fitting problems because it tries to approximate the target output that in a classification problem is only a codification of the classes not a value we try to fit. This idea of using regression methods for resolving qualitative problems is not new $[15,16]$, and in [17] it is shown that MLF neural networks trained with the backpropagation learning rule for classification problems exhibited a similar performance to optimal discriminant methods dealing with normal multivariate distributed data sets.

As a supervised learning rule, backpropagation needs a way to measure the difference between the output obtained for the network and the target output. So an error function has to be defined for looking for a minimum in the error hyper-surface. The most usual error function is the squared scaled euclidean distance between the output and the target values.

Based on the calculated error, the delta term is computed and backpropagated through the network correcting the weights and starting again until some goals are reached. Here, several questions need to be taken into account before and during training: a correct initialization of the weights must be chosen, an adequate learning rate for controlling the stepsize of change of the weights, maybe a momentum term, minimization method for moving along the error hypersurface, etc.

Another question to consider is the overtraining effect, i.e. the effect in which the network uses its degrees of freedom to store the individual patterns instead of learning the underlying features [5]. Such a network is good at recognizing the patterns used for learning but has lost its generalization ability, that is to say its ability to respond correctly to other structurally equal inputs but which differ from the patterns for random reasons (experimental variability, etc.).

\section{Experimental and results}

\subsection{Data}

One of the goals of the paper is to compare the classification ability between MLF and RBF neural networks. So, the authors decided to simulate the data sets used for the training of the networks. This is above all to control the distribution of the data, to be sure the training and test sets are exactly in the same input space and to avoid any other additional and 
probably unknown problems that can appear with real data sets and can mask some properties of the networks.

Taking into account the differences between a discriminant and a modelling technique and in order to evaluate the quality of the results, it was decided to use data sets with "a priori" established values for the sensibility and specificity. In this way one can obtain some conclusions about the performance of both kinds of networks.

Specificity of a class depends basically on the separation between class models so depends on the criterion used for computing the class boundaries. As in fact the class boundaries are represented by the critical distance, sensibility and specificity depend on the distance used.

According to [4], using the Euclidcan distance, $D$, and $p$ and $p^{\prime}$ being the values for sensibility and specificity respectively, $D\left(p, p^{\prime}\right)$ can be expressed as a linear combination of non-integer powers of the number of variables in the problem.

At $95 \%$ confidence level, thus considering $\alpha=\beta$ $=0.05$, the theoretical values for sensibility $(1-\alpha)$ and specificity $(1-\beta)$ are both equal to 0.95 .

If $n$ represents the number of variables in the problem, the corresponding theoretical distance between models for $p=p^{\prime}=0.95$ can be calculated by means of the following formula [4]:

$$
\begin{aligned}
D(0.95,0.95)= & 2.80589+0.89441 \sqrt{n}-0.08390 n \\
& +0.00465 n \sqrt{n}
\end{aligned}
$$

Based on this formula, three independent normal multivariate data sets with objects belonging to two classes were generated in a 5-dimensional space. All of them with theoretical values for sensibility and specificity equal to 0.95 . These three sets were: (i) a training set to train the network, with 50 objects in each class; (ii) a monitoring set, also with 100 objecls (50 per class) and dedicated to control ("to monitor") the prediction behaviour of the networks during learning to avoid the overtraining effect; (iii) a test set with 200 objects ( 100 per class) in order to test the prediction ability of the networks.

The objects are characterized by five uncorrelated variables. Each variable in class 1 follows a normal distribution with zero mean and variance equal to one, whereas in class 2 the mean is $D / \sqrt{5}$ and the vari- ance is one, with $D$ according to Eq. (4) for $n=5$. So, the classes are defined by their centroid: $(0, \ldots, 0)$ for class 1 and $([D / \sqrt{5}], \ldots,[D / \sqrt{5}])$ for class 2 .

When the networks are used for classification tasks, binary target outputs can be selected. In this way, the output of the network should give the class of the object presented to the network. Among all the possible codifications, each class was associated with only one output unit. This is in order to simplify the interpretation of the network outputs and to avoid an implicit sort of ordering in the classes [18]. So the codification of the classes was $(1,0)$ for class 1 and $(0,1)$ for class 2 .

\subsection{Neural networks}

Based on the different ways the networks operate, only the number of units in the input and output layers was previously fixed (five and two units, respectively). The final topology of the networks, i.e. number of hidden units, was decided during training in the sense that the training procedure checked the results with different configurations before choosing the final one.

\subsubsection{Structure of the networks}

In practice, if $\mathbf{x}=\left(x_{1}, x_{2}, \ldots, x_{5}\right)$ represents an object in the five dimensional input space, " $n_{\text {hid }}$ " is the number of hidden units in the network, $\lambda_{j k}$ are the weights connecting the various kernels with the output unit and $\mathbf{O}=\left(O_{1}, O_{2}\right)$ is the output of the network, we can write:

(i) For an MLF neural network that uses as transfer functions in its hidden layer those defined by Eq. (1)

$$
O_{k}=\sum_{j=1}^{n_{\mathrm{hid}}} \lambda_{j k} \cdot \psi_{j}\left(\sum_{i=1}^{5} w_{i j} x_{i}\right)
$$

where $w_{i j}$ represents the connection weight between the $i$ th unit in the input layer and the $j$ th hidden unit.

(ii) For an RBF neural network that uses as radial functions in its hidden layer those defined by Eq. (3)

$O_{k}=\sum_{j=1}^{n_{\text {hid }}} \lambda_{j k} \cdot \psi_{j}\left(\left\|c_{j}-x\right\|_{2}\right)$

where $c_{j} \in \mathbb{R}^{5}$ represents the centroid of the $j$ th kernel function. 
Then, the error is computed as the difference between the output obtained by the network, $\mathbf{O}$, and the target output, $\mathbf{T}=\left(T_{1}, T_{2}\right)$, based on the following error function:

$E=\frac{1}{2} \sum_{j=1}^{2}\left(O_{k}-T_{k}\right)^{2}$

\subsubsection{Training}

A lot of network parameters (weights) need to be trained. In addition to the generic problems concerning the training of neural networks with the backpropagation learning rule, some aspects need to be considered when training RBF networks:

(i) The initialization of the centroids of the kernel functions. If these centroids are chosen too far from the input range, the output of the RBF-unit becomes zero almost independently of the initial widths. This results in very long training and probably the network will not be able to find the correct parameters. There are several methods to find the initial centroids of the radial base functions in the range of a class in the input space, for example by means of the $k$-means clustering technique (for example [19]), or using a Kohonen learning rule [20] or by means of a genetic algorithm (e.g. [21]). All of them are unsupervised techniques that place the centroid among the centroids of a certain cluster. Therefore the initial centroids of the radial base functions are better chosen around the real centroids of the input data, if they are known.

(ii) The width factors. In fact, the learning of the network is done by adjusting the size of $S=\sigma^{2}$ (not of $\sigma$ ) in the modified Gaussian kernel function [Eq. (3)]. So for mathematical reasons this value must not become zero nor negative during training. A precaution for this problem must be taken.

(iii) The initialization of the $S$ factors. Even if the centroids of the kernel functions are in the correct range, the behaviour of the network depends on the $S$ factors. If they are chosen too large, the responses will become one for every object and the training time will become very long. If they are chosen too small, the responses will become zero for every object and we end up in the same situation as (i). So a kind of compromise between the centroids of the radial base functions and their widths has to be found.

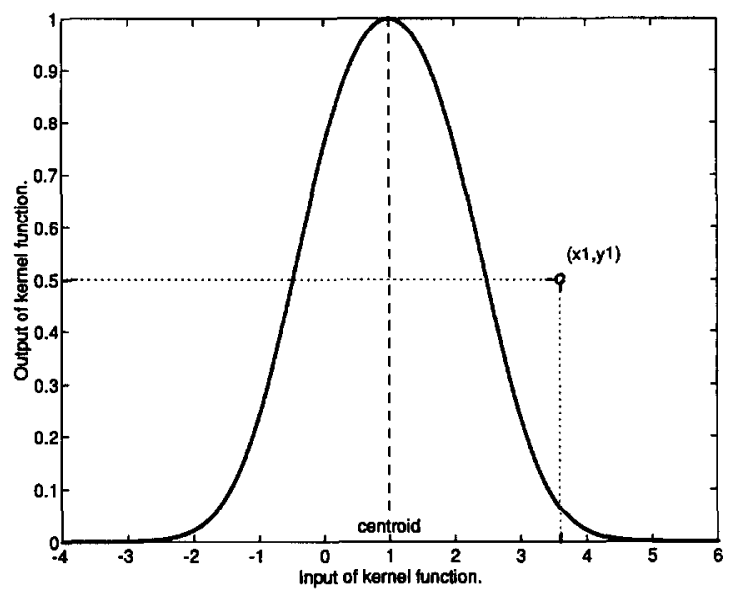

Fig. 2. Radial base function in one dimension: effect of moving the centroid and the width of the kernel function to obtain the target value $y_{1}$ for the corresponding input $x_{1}$.

(iv) Related to the last questions, the same situation is likely to occur during training. There are two ways of adapting the weights of the hidden layer: the centroid of the kernel function can be adjusted or the $S$ factor can be adjusted (see Fig. 2). It has been found that when larger networks are trained, the factor $S$ tends to drift towards very low values. This is usually an indication that the data are linearly separable. As in (i) there are several ways to tackle this question. One of them is to train separately the centres by means of clustering techniques, estimating the local densities for each kernel, ...- and then fix them and train the rest of the parameters. Maintaining the idea of using the backpropagation algorithm to train simultaneously all the weights involved in the network, a way to counter this behaviour without modifying the simultaneous algorithm is to introduce an extra smaller learning rate for the $S$ factor. In this way the adaption of the centroid is favoured.

(v) The delta term used for updating the weights is computed based on the direction shown by the steepest gradient descent method. For MLF networks the direction shown is the direction in which the derivative of the function respect to the weights $w_{i j}$ is negative. For RBF networks, using the same error function, the network parameters have a different meaning and they are adjusted independently but simultaneously. So there are two, not necessarily equal, directions shown by the method: one computed based 
on the derivative of the function with respect to the centroids of the kernel functions, $c_{i j}$, and another based on the derivative with respect to the $S_{j}$ (the $S$ factor associated with the $j$ th kernel function).

\subsection{Replication of the networks}

Both MLF and RBF were trained with the same procedure. For MLF all the weights were initialized randomly using the Nguyen-Widrow initialization method which is implemented in the Neural Network Toolbox [22] from MATLAB. For RBF, due to the different meaning of the hidden units and the bigger problems with the initialization of their weights, the initialization was different: the parameters associated with the output layer were initialized with random normal values with zero mean and standard deviation of one; the centroids of the radial base functions were initialized near the theoretical class centroids; all the widths were initialized constantly equal to 0.5 ; and $R$ was fixed at 1 , because this value exhibited the best performance.

As with other flexible estimation techniques, it is desirable to allow the structural parameters of the networks to be chosen based at least partly on the data [23]. The topology selection problem can be addressed in a variety of ways. In this paper we used a monitoring set approach [5] to model selection, stopping the training when the error on the monitoring set reaches a minimum. So, various attempts with different numbers of hidden units were checked to find out the best topology of the network. In every case, the monitoring set was used as stopping criterion to avoid the overtraining effect, based on the best prediction ability of the networks during training.

The best results for MLF networks were obtained with one hidden unit. For RBF networks, despite the fact that, due to the structure of the data sets and the way the networks operate, onc hidden unit per class should perform the classification task, in fact this was not the case. When only two hidden units (one unit per class) are used, the networks exhibited a very bad performance. The best results were obtained with six hidden units. When more hidden units are used, the network has a better performance but also the training time increases rapidly.

To study the stability and reproducibility of the networks, ten different networks of each type were independently trained with the use of the same data set.

The twenty networks were trained based on the percentage of correct classifications on the monitoring set and, also taking into account the behaviour of the error on this monitoring set during training. When the percentage of correct classifications started to decrease and the error to increase, the training was considered finished.

The training was implemented in batch mode, i.e. all the objects belonging to the training set were presented to the network simultaneously. In this way, the dependency of the delta learning rule to the presentation of the objects was avoided. But, in this case, the error function should take into account the error for all the objects.

If " $n_{\mathrm{obj}}$ " represents the number of objects in the data matrix, each one has its own output, $O_{p k}$, and target, $T_{p k}$, and Eq. (7) becomes:

$E=\sqrt{\frac{1}{2 n_{\mathrm{obj}}} \sum_{p=1}^{n_{\mathrm{obj}}} \sum_{k=1}^{2}\left(O_{p k}-T_{p k}\right)^{2}}$

Then percentages of correct classifications for the training and test sets were computed. The results obtained are listed in Tables 1 and 2, together with calibration error (value of Eq. (8) with the outputs obtained by applying the networks on the training set)

Table 1

Percentage of correct classifications for RBF networks. \% CC is the percentage of correct classifications on the referenced set. The last row contains the mean values for the ten networks

\begin{tabular}{llll}
\hline $\begin{array}{l}\text { Calibration } \\
\text { error }\end{array}$ & $\begin{array}{l}\text { Prediction } \\
\text { error }\end{array}$ & $\begin{array}{l}\% \mathrm{CC} \\
\text { training }\end{array}$ & $\begin{array}{l}\text { \% CC } \\
\text { test }\end{array}$ \\
\hline 0.1351 & 0.1364 & 98 & 98.0 \\
0.1375 & 0.1368 & 96 & 98.5 \\
0.1588 & 0.1629 & 96 & 96.0 \\
0.1383 & 0.1423 & 97 & 98.5 \\
0.1387 & 0.1386 & 98 & 97.5 \\
0.1352 & 0.1463 & 98 & 97.5 \\
0.1451 & 0.1437 & 98 & 98.5 \\
0.1260 & 0.1276 & 98 & 98.5 \\
0.1258 & 0.1304 & 98 & 98.5 \\
0.1257 & 0.1313 & 98 & 98.5 \\
\hline 0.1366 & 0.1396 & 97.5 & 98.0 \\
\hline
\end{tabular}


Table 2

Percentage of correct classifications for MLF networks. \% CC is the percentage of correct classifications on the referenced set. The last row contains the mean values for the ten networks

\begin{tabular}{llll}
\hline $\begin{array}{l}\text { Calibration } \\
\text { error }\end{array}$ & $\begin{array}{l}\text { Prediction } \\
\text { error }\end{array}$ & $\begin{array}{l}\text { \% CC } \\
\text { training }\end{array}$ & $\begin{array}{l}\text { \% CC } \\
\text { test }\end{array}$ \\
\hline 0.0373 & 0.0769 & 100 & 100 \\
0.0386 & 0.0771 & 100 & 100 \\
0.0378 & 0.0770 & 100 & 100 \\
0.0384 & 0.0771 & 100 & 100 \\
0.0365 & 0.0768 & 100 & 100 \\
0.0370 & 0.0769 & 100 & 100 \\
0.0375 & 0.0769 & 100 & 100 \\
0.0369 & 0.0769 & 100 & 100 \\
0.0365 & 0.0768 & 100 & 100 \\
0.0384 & 0.0771 & 100 & 100 \\
\hline 0.0375 & 0.0770 & 100 & 100 \\
\hline
\end{tabular}

and prediction error (value of Eq. (8) with the outputs of the test set). As can be seen in the tables and, regarding to the errors and percentages of correct classifications, MLF networks perform better than RBF networks. Anyway, the networks are very reproducible and quite stable.

\subsection{Structural similarity of the different indepen- dently trained networks on the same data set}

We have ten RBF networks and ten MLF networks with similar performance and similar values for percentage of correct classifications in both classes but with different weights. As, in fact, a network is determined by the values of its weights, we have ten different networks of each type that solve the problem in a similar way. The training of the network consists of looking for a minimum in an unknown error surface, so obviously the solution is not necessarily unique. However, there should be some common characteristics for all networks because they are solving exactly the same problem and with the same topology.

For our purposes, we will describe RBF networks by three matrices: $\mathrm{C}, \mathrm{S}$ and $\mathrm{W}$, which will store the parameters associated with the hidden ( $C$ and $S$ ) and the output layer (W). This means that in $C$ we have stored the values for the centroids of the radial base functions, $c_{i}$, and in $S$ the squared widths of the kernel functions, $S_{i}$, whereas in W the parameters $\lambda_{j k}$ are stored. In this sense, the $i$ th column of $\mathrm{C}$ contains the coordinates of the centroid of the $i$ th-kernel function, $c_{i}$, and the $i$ th column in $S$ stores the corresponding $S_{i}$ factor. W contains the coefficients for the final linear combination of kernel functions, one column per output there are in the problem.

In our case, the RBF networks have 6 hidden units, five inputs and two outputs, so the size of $\mathrm{C}$ is $\mathbf{5}$ by 6 , of $S$ is 1 by 6 and of $W$ is 6 by 2 .

As the structure of the weights and their meaning are clear, in fact, we can define each network by only one matrix, $\mathrm{N}$, which consists of joining vertically $\mathrm{C}$, $\mathrm{S}$ and the transpose of $\mathrm{W}$. Thus $\mathrm{N}$ is an 8 by 6 matrix that stores in each of their columns one centroid, the corresponding squared width and the corresponding parameters (one per class) of the final linear layer.

In this way, we have ten different matrices, $\mathrm{N}_{i}(i$ $=1,2, \ldots, 10$ ), each one defining an RBF network. If we superimpose all of them, we have a so-called three-way data set (three-dimensional array), $\mathrm{Z}$, which contains the information of all networks. We are interested in studying the information provided by $\mathrm{Z}$.

If we imagine $\mathrm{Z}$ as a parallelepiped in the threedimensional space and call $I, J, K$ the three axes involved in the space (as if it were a three-dimensional system of coordinates) with $I=\{1,2, \ldots, 10\}, J=\{1$, $2, \ldots, 6\}$ and $K=\{1,2, \ldots, 8\}$ we have, from the mathematical point of view, a mapping $\mathrm{Z}: \mathrm{I} \times \mathrm{J} \times \mathrm{K}$ $\rightarrow \mathbb{R}$ that assigns to each triple $(i, j, k) \in \mathrm{I} \times \mathrm{J} \times \mathrm{K}$ the observation $z_{i j k}$.

To handle this three-dimensional array, we reduced it to a data table by so called unfolding. With this unfolded matrix, one tries to obtain some conclusions by analysing it by principal component analysis (PCA).

There are three possibilities of unfolding the matrix $\mathrm{Z}$ [24]. In each situation, one of the modes remains unchanged and the other two are combined to yield a single one. In this way, we obtain three twodimensional matrices, $\mathrm{Z}_{i}, \mathrm{Z}_{j}$ and $\mathrm{Z}_{k}$ where the index refers to the mode that remains unchanged.

More specifically, the matrices are:

(1) $Z_{i}$ consists of a matrix 10 by 48 made by choosing in $\mathrm{Z}$ the planes parallel to the IK-plane (J- 
slices), transposing them and joining them horizontally (i.e. putting them next to each other). This way, in the rows of the matrix appear the different networks:

$\left(\begin{array}{ccc}c_{1} S_{1} \lambda_{11} \lambda_{12} & \ldots & c_{6} S_{6} \lambda_{61} \lambda_{62} \\ & \ldots & \\ c_{1} S_{1} \lambda_{11} \lambda_{12} & \ldots & c_{6} \ldots \lambda_{62}\end{array}\right)$

(2) $\mathrm{Z}_{j}$ consists of a 6 by 80 matrix made by choosing in $Z$ the planes parallel to the IJ-plane (Kslices), transposing them and joining them horizontally:

$$
\left(\begin{array}{rlc}
c_{1} S_{1} \lambda_{11} \lambda_{12} & \ldots & c_{1} S_{1} \lambda_{11} \lambda_{12} \\
& \ldots & \\
c_{6} S_{6} \lambda_{61} \lambda_{62} & \ldots & c_{6} S_{6} \lambda_{61} \lambda_{62}
\end{array}\right)
$$

(3) $\mathrm{Z}_{k}$ consists of an 8 by 60 matrix made by choosing in $\mathrm{Z}$ the planes parallel to the JK-plane (Islices), transposing them and joining them horizontally:

$$
\left(\mathrm{N}_{1} \mathrm{~N}_{2} \ldots \mathrm{N}_{10}\right)
$$

Following the Tucker 1 method [25] separate PCA analyses were performed for each data matrix obtained by the different ways of unfolding. Then, ignoring the loadings of combination models and saving merely the scores of the respective isolated mod-

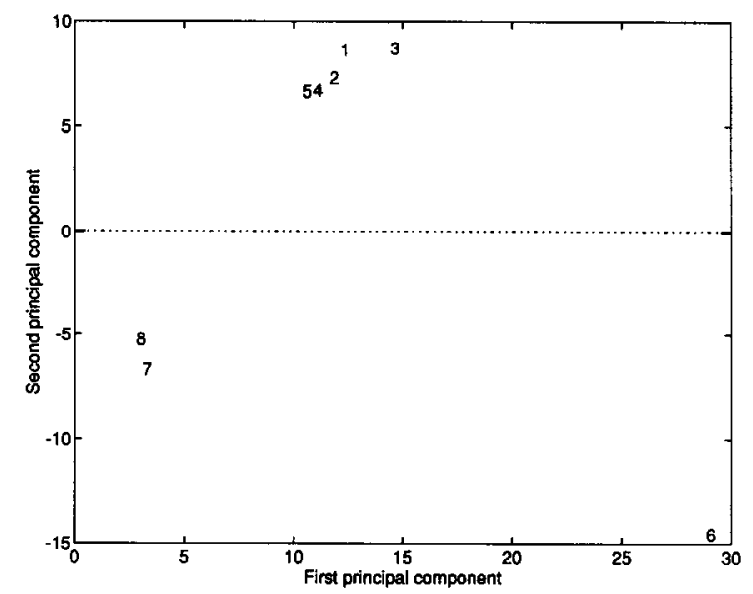

Fig. 3. Plot the scores of $Z_{k}$ unfolding in the plane formed by the first two eigenvectors.

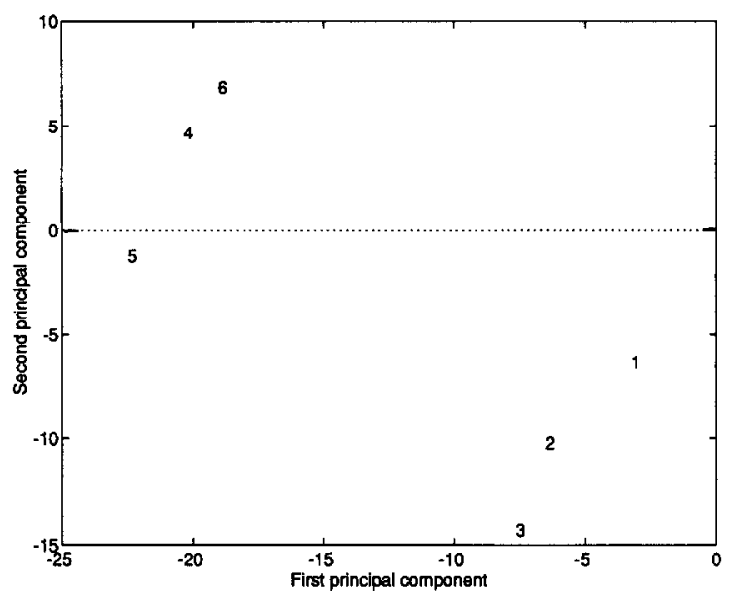

Fig. 4. Plot the scores of $Z_{j}$ unfolding in the plane formed by the first two eigenvectors.

els, one obtains separate PCA representations for the items of each mode.

Figs. 3-5 plot the scores in the plane formed by the first two eigenvectors and for the three ways of unfolding.

For $Z_{k}$ the first two eigenvectors explain $73.61 \%$ of the variance. From Fig. 3 it can be deduced that these two eigenvectors separate rows $1-5$ (which in $\mathrm{Z}_{k}$ contain the class centroids of the kernel functions) from row 6 (the $S$ factors) and from rows 7-8 (which contain the coefficients of the output layer). This structure coincides with the structure of the matrices $\mathrm{N}_{i}$ joined to form $\mathrm{Z}_{k}$.

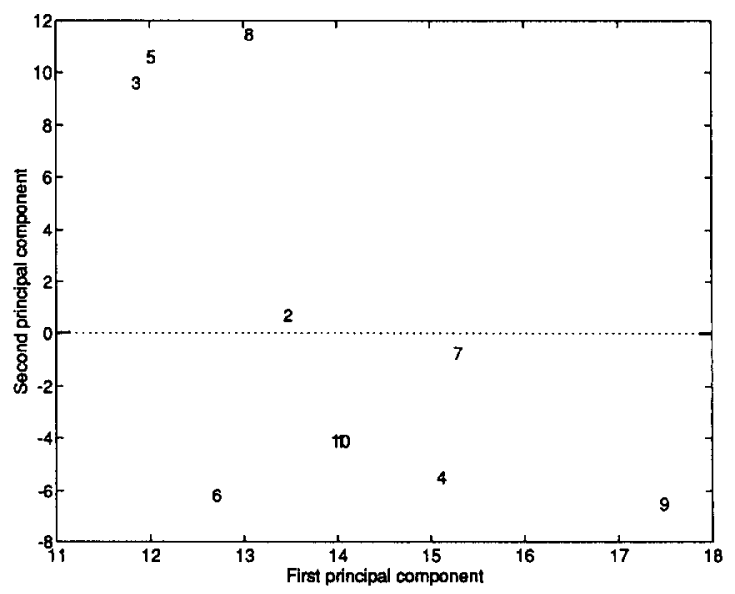

Fig. 5. Plot the scores of $Z_{i}$ unfolding in the plane formed by the first two eigenvectors. 
For $Z_{j}$ the first two eigenvectors explain $64.69 \%$ of the variance. From Fig. 4 it can be deduced that these two eigenvectors separate the first three rows of $\mathrm{Z}_{j}$ from the last three rows. In the structure of $\mathrm{Z}_{j}$ each row corresponds with each centroid (of the kernel functions) and its associated parameters. In all the networks, the first three kernel functions are modelling class 1 and the last three class 2 , so this is what we see in this plot.

For $\mathrm{Z}_{i}$ the first two eigenvectors explain $84.60 \%$ of the variance and Fig. 5 does not show any special structure in the disposition of the scores. As here the rows of $\mathrm{Z}_{i}$ represent the different networks, we can say that there are not significant differences between them, that is, from among the numerical values of the parameters, they are structurally equal.

These analyses confirm what one can expect as regards the behaviour of the networks and the structure of the different ways of unfolding.

For MLF networks, a similar analysis does not have such a clear interpretation, because the MLFweights have no physical meaning and, in contrast with RBF, MLF is not a local method, so, the information is distributed globally in the network.

\subsection{Discrimination and modelling}

In this paper, RBF and MLF networks are trained to classify objects into two different classes. When an object belongs to class 1 the network should produce an output of $(1,0)$ and an object of class 2 should produce an output of $(0,1)$. But, in fact, these values are not obtained because of the linear output of the networks. Instead, the network produces values that spread around zero and one. So, the user must interpret the output of the network.

Different classification criteria can be used for the interpretation of the output. The most usual one is based only on discrimination and consists of defining a threshold valuc to assign each output of the network either to one or zero. Results shown in Tables 1 and 2 were computed based on this criterion using 0.5 as threshold value.

In class modelling techniques there are two questions to consider: (i) the way the model is constructed and (ii) the decision rule used for assigning an object to a class (closed box).

The class models are defined with the outputs ob- tained with the training set. In this case, the models consist of a class centroid and a critical distance (that acts as a class boundary). The class centroids were calculated as the mean values of the outputs for class 1 and class 2 separately. The class boundary can be defined based on the similarities (or on the dissimilarities) among the objects in that class. In this paper, the distance between each object in a class and its class centroid was computed, so fifty values of the distances were obtained for each class. From the distribution of these values, a critical value for the distance was computed. In this way, we have two class centroids, $c_{1}$ and $c_{2}$, and two critical distances, $d_{\text {crit }}$ and $d_{\text {crit }_{2}}$.

The decision rule for the classification of an object in a class is then made with the critical distance. If the distance between an object in the output space and $c_{1}$ is less than $d_{\text {crit }_{1}}$ then the object is assigned to class 1 . If the distance between an object in the output space and $c_{2}$ is less than $d_{\text {crit }_{2}}$ then the object is assigned to class 2 .

To calculate the critical distance, a value of the probability is needed to express the percentage of objects in the training set that belong to a certain class. As the value for $\alpha$ was a priori fixed at 0.05 , this value was chosen. In this way, $95 \%$ of the objects are within the class model (that means $95 \%$ of the distances computed between the object and the class centroid are smaller than the corresponding critical distance).

Among the possibilities for calculating the critical distance, in this paper the following were considered:

\subsubsection{Euclidean distance with $95 \%$ sample per- centile (so $1-\alpha$ probability)}

To construct the model, the euclidean distance between each output obtained for the network with the training set and the corresponding class centroid is calculated. The critical distance is the value for which the cumulative distribution function is equal to $95 \%$, the $95 \%$ sample percentile. To determine [2] this sample percentile, the fifty distances per class are ordered in increasing order giving a vector $\mathbf{d}$ of distances. The critical distance is the value whose position fits the equation:

$d_{\text {crit }}=\mathbf{d}(47)+0.5[\mathbf{d}(48)-\mathbf{d}(47)]$

so the corresponding point obtained by interpolating the distribution function to $95 \%$. 
Table 3

Class models (class centroids and critical distances), sensibility and specificity for RBF networks. The last row contains the mean values for the ten networks. Euclidean distance

\begin{tabular}{lcllllll}
\hline Centroid class 1 & Centroid class 2 & $d_{\text {crit }}$ & $d_{\text {crit }}$ & $\begin{array}{l}\text { Sensibility } \\
\text { class } 1\end{array}$ & $\begin{array}{l}\text { Specificity } \\
\text { class 1 }\end{array}$ & $\begin{array}{l}\text { Sensibility } \\
\text { class 2 }\end{array}$ & $\begin{array}{l}\text { Specificity } \\
\text { class 2 }\end{array}$ \\
\hline$(0.9382,0.0157)$ & $(0.0808,0.9745)$ & 0.2813 & 0.3861 & 95 & 100 & 93 & 100 \\
$(0.9642,0.0204)$ & $(0.0098,0.9044)$ & 0.3258 & 0.3619 & 95 & 100 & 95 & 100 \\
$(0.9627,0.0312)$ & $(-0.0061,0.8535)$ & 0.3456 & 0.3761 & 94 & 100 & 92 & 100 \\
$(0.8852,0.0160)$ & $(0.0262,0.9208)$ & 0.2357 & 0.3562 & 96 & 100 & 92 & 100 \\
$(0.9628,0.0268)$ & $(0.0060,0.9124)$ & 0.3323 & 0.3508 & 94 & 100 & 92 & 100 \\
$(0.9726,0.0276)$ & $(0.0039,0.9089)$ & 0.3107 & 0.3618 & 97 & 100 & 88 & 100 \\
$(0.9703,0.0409)$ & $(0.0091,0.8474)$ & 0.3023 & 0.3447 & 97 & 100 & 95 & 100 \\
$(0.9644,0.0117)$ & $(0.0183,0.9981)$ & 0.3152 & 0.3832 & 96 & 100 & 95 & 100 \\
$(0.9583,0.0199)$ & $(0.0233,0.9663)$ & 0.2855 & 0.3823 & 96 & 100 & 94 & 100 \\
$(0.9677,0.0199)$ & $(0.0207,0.9628)$ & 0.2886 & 0.3841 & 96 & 100 & 93 & 100 \\
& & 0.3023 & 0.3687 & 95.6 & 100 & 92.9 & 100 \\
\hline
\end{tabular}

Once the model is defined, the test set is presented to the networks and the euclidean distance between each output and both class centroids is computed. With the decision rule described before, the sensibility and specificity can be obtained for both classes on the test set. Tables 3 and 4 show the class models (class centroid and critical distance constructed with the training set independently for class 1 and class 2), sensibility and specificity for both classes (applying the network on the test set) and for the ten networks.

It can be seen from the tables that there is a good reproducibility of both RBF and MLF networks and there is no significant difference between the ob- tained models with regard to sensibility and specificity.

Considering that the class centroids of the models were computed as the mean values of the outputs obtained for the network on the training set, the class centroids found are really close to the theoretical class centroids in the output space, $(1,0)$ and $(0,1)$. Looking at the boundaries of the models (critical distances) which contain inside $95 \%$ of the output values, it is evident that in every case, these outputs for objects in class 1 form a cluster around the target output $(1,0)$ and for class 2 form a cluster around the target output $(0,1)$. The $\mathrm{RBF}$ values are more spread out than the values obtained with MLF networks, that

Table 4

Class models (class centroids and critical distances), sensibility and specificity for MLF networks. The last row contains the mean values for the ten networks. Euclidean distance

\begin{tabular}{lcllllll}
\hline Centroid class 1 & Centroid class 2 & $d_{\text {crit }_{1}}$ & $d_{\text {crit }_{2}}$ & $\begin{array}{l}\text { Sensibility } \\
\text { class 1 }\end{array}$ & $\begin{array}{l}\text { Specificity } \\
\text { class 1 }\end{array}$ & $\begin{array}{l}\text { Sensibility } \\
\text { class 2 }\end{array}$ & $\begin{array}{l}\text { Specificity } \\
\text { class 2 }\end{array}$ \\
\hline$(0.9972,0.0027)$ & $(0.0027,0.9972)$ & 0.1253 & 0.0963 & 98 & 100 & 93 & 100 \\
$(0.9970,0.0029)$ & $(0.0029,0.9970)$ & 0.1303 & 0.0994 & 98 & 100 & 93 & 100 \\
$(0.9971,0.0028)$ & $(0.0028,0.9971)$ & 0.1274 & 0.0976 & 98 & 100 & 93 & 100 \\
$(0.9970,0.0029)$ & $(0.0029,0.9970)$ & 0.1294 & 0.0989 & 98 & 100 & 93 & 100 \\
$(0.9973,0.0026)$ & $(0.0026,0.9973)$ & 0.1223 & 0.0943 & 98 & 100 & 93 & 100 \\
$(0.9972,0.0027)$ & $(0.0027,0.9972)$ & 0.1245 & 0.0957 & 98 & 100 & 93 & 100 \\
$(0.9971,0.0028)$ & $(0.0028,0.9971)$ & 0.1261 & 0.0967 & 98 & 100 & 93 & 100 \\
$(0.9972,0.0027)$ & $(0.0027,0.9972)$ & 0.1239 & 0.0953 & 98 & 100 & 93 & 100 \\
$(0.9973,0.0026)$ & $(0.0026,0.9973)$ & 0.1226 & 0.0945 & 98 & 100 & 93 & 100 \\
$(0.9970,0.0029)$ & $(0.0029,0.9970)$ & 0.1296 & 0.0990 & 98 & 100 & 93 & 100 \\
& & 0.1261 & 0.0968 & 98 & 100 & 93 & 100 \\
\hline
\end{tabular}


Table 5

Class models (class centroids and critical distances), sensibility and specificity for RBF networks. The last row contains the mean values for the ten networks. Mahalanobis distance

\begin{tabular}{lcllllll}
\hline Centroid class 1 & Centroid class 2 & $d_{\text {crit }_{1}}$ & $d_{\text {crit }_{2}}$ & $\begin{array}{l}\text { Sensibility } \\
\text { class 1 }\end{array}$ & $\begin{array}{l}\text { Specificity } \\
\text { class 1 }\end{array}$ & $\begin{array}{l}\text { Sensibility } \\
\text { class 2 }\end{array}$ & $\begin{array}{l}\text { Specificity } \\
\text { class 2 }\end{array}$ \\
\hline$(0.9382,0.0157)$ & $(0.0808,0.9745)$ & 2.9293 & 2.9610 & 95 & 100 & 94 & 100 \\
$(0.9642,0.0204)$ & $(0.0098,0.9044)$ & 2.9156 & 2.9674 & 96 & 100 & 95 & 100 \\
$(0.9627,0.0312)$ & $(-0.0061,0.8535)$ & 2.7761 & 2.6060 & 94 & 100 & 91 & 100 \\
$(0.8852,0.0160)$ & $(0.0262,0.9208)$ & 2.2332 & 2.9447 & 96 & 100 & 93 & 100 \\
$(0.9628,0.0268)$ & $(0.0060,0.9124)$ & 2.8793 & 2.8085 & 94 & 100 & 94 & 100 \\
$(0.9726,0.0276)$ & $(0.0039,0.9089)$ & 2.8038 & 2.9314 & 94 & 100 & 90 & 100 \\
$(0.9703,0.0409)$ & $(0.0091,0.8474)$ & 2.8923 & 2.9592 & 94 & 100 & 96 & 100 \\
$(0.9644,0.0117)$ & $(0.0183,0.9981)$ & 2.7925 & 2.9889 & 95 & 100 & 95 & 100 \\
$(0.9583,0.0199)$ & $(0.0233,0.9663)$ & 2.7800 & 2.9023 & 96 & 100 & 94 & 100 \\
$(0.9677,0.0199)$ & $(0.0207,0.9628)$ & 2.6889 & 2.9235 & 95 & 100 & 94 & 100 \\
& & 2.7691 & 2.8993 & 94.9 & 100 & 93.6 & 100 \\
\hline
\end{tabular}

is, for MLF networks the clusters are smaller than for RBF networks, which is what one expects for the way the networks operate.

The input space was constructed with $95 \%$ sensibility and specificity. The models obtained with the output of the networks show a too high value for the specificity $(100 \%)$ whereas the sensibility is always higher for class 1 (and greater than the theoretical sensibility) than for class 2 (always less than the theoretical value). Also, for MLF networks the models have larger values for the sensibility in both classes than for RBF networks. These results correspond to what one could expect because of the "size" of the clusters. Anyway, the results are not very different and almost stable for all networks.

\subsubsection{Mahalanobis distance with $95 \%$ sample per- centile}

The model is constructed as in the last section, but using the Mahalanobis distance instead of the euclidean distance. In this way, the correlation among the variables is taken into account and the model fol- lows the direction shown for the outputs of the networks. Tables 5 and 6 show the class models, sensibility and specificity computed with this criterion and for both RBF and MLF networks. For MLF networks, only the results of one of the networks are shown, because only this result is reliable. For the calculation of the Mahalanobis distance an inverse variance-covariance matrix needs to be calculated. In the other ninc networks this matrix is close to a singular matrix so the results may be inaccurate.

Also in this case, the models are quite reproducible and there is no significant difference between the obtained models related to sensibility and specificity.

The class centroids of the models are, of course, the same. For the sensibility, the only reliable result for MLF shows the same performance as for section 3.5.1. This is because the clusters are almost spherical and very close to the class centroid, so the euclidean distance is equal to the Mahalanobis distance. For RBF networks, the models are better in both classes related to the sensibility. This is because

Table 6

Class models (class centroids and critical distances), sensibility and specificity for MLF networks. The last row contain the mean values for the ten networks. Mahalanobis distance

\begin{tabular}{llllllll}
\hline Centroid class 1 & Centroid class 2 & $d_{\text {crit }}$ & $d_{\text {crit }_{2}}$ & $\begin{array}{l}\text { Sensibility } \\
\text { class 1 }\end{array}$ & $\begin{array}{l}\text { Specificity } \\
\text { class 1 }\end{array}$ & $\begin{array}{l}\text { Sensibility } \\
\text { class 2 }\end{array}$ & $\begin{array}{l}\text { Specificity } \\
\text { class 2 }\end{array}$ \\
\hline$(0.9972,0.0027)$ & $(0.0027,0.9972)$ & 2.0065 & 2.1797 & 98 & 100 & 93 & 100 \\
\hline
\end{tabular}


they are following the direction of the spread of the values. But, even in this case, the models for both RBF and MLF networks have a specificity of $100 \%$.

\subsection{Robustness of the trained networks}

So far, we have studied properties about the performance of the networks both as discriminating and as modelling techniques. Now we are interested in the sensitivity of the networks to deviations in the input space, in other words, in the robustness of the networks obtained. These deviations are likely to occur in practice, the measurements in a laboratory, for instance, are not exact and for future predictions it is not guaranteed that the data will have the same structure than the data used for training the networks. The problem posed now is the behaviour of the networks when predicting new data that can vary with respect to those data used to train the networks. This question is central in all the inference procedures because, in practice, one will deal with unexpected and unknown deviations. So, further to the noise in the training data sets, we consider an additional source of noise, probably unknown and different, which is distorting the expected noise taken into account when training the networks.

As the networks are nonparametric inference procedures, we followed a scheme similar to those used to evaluate the robustness of nonparametric statistical procedures: Monte Carlo simulations were performed to obtain some information about the effect of noise to the networks and about the amount of noise the networks can support. On the other hand, the errors in the input space are usually assumed to be normally distributed but in practice this assumption does not always hold. Thus, a study of the robustness of the networks to different types of noise in the input space was also performed.

Three different kinds of noise were used: noise following (i) a normal distribution, (ii) a uniform distribution and (iii) a Laplace distribution, all of them with zero mean.

These distributions are commonly used to study the efficiency of non-parametric and robust statistical procedures [26]. These distributions show the different situations which might occur in practice:

(i) Noise due to errors with the highest probability in its central part, due for instance to errors which different sign that are "compensating" with each other. See the probability density function plotted in Fig. 6a.
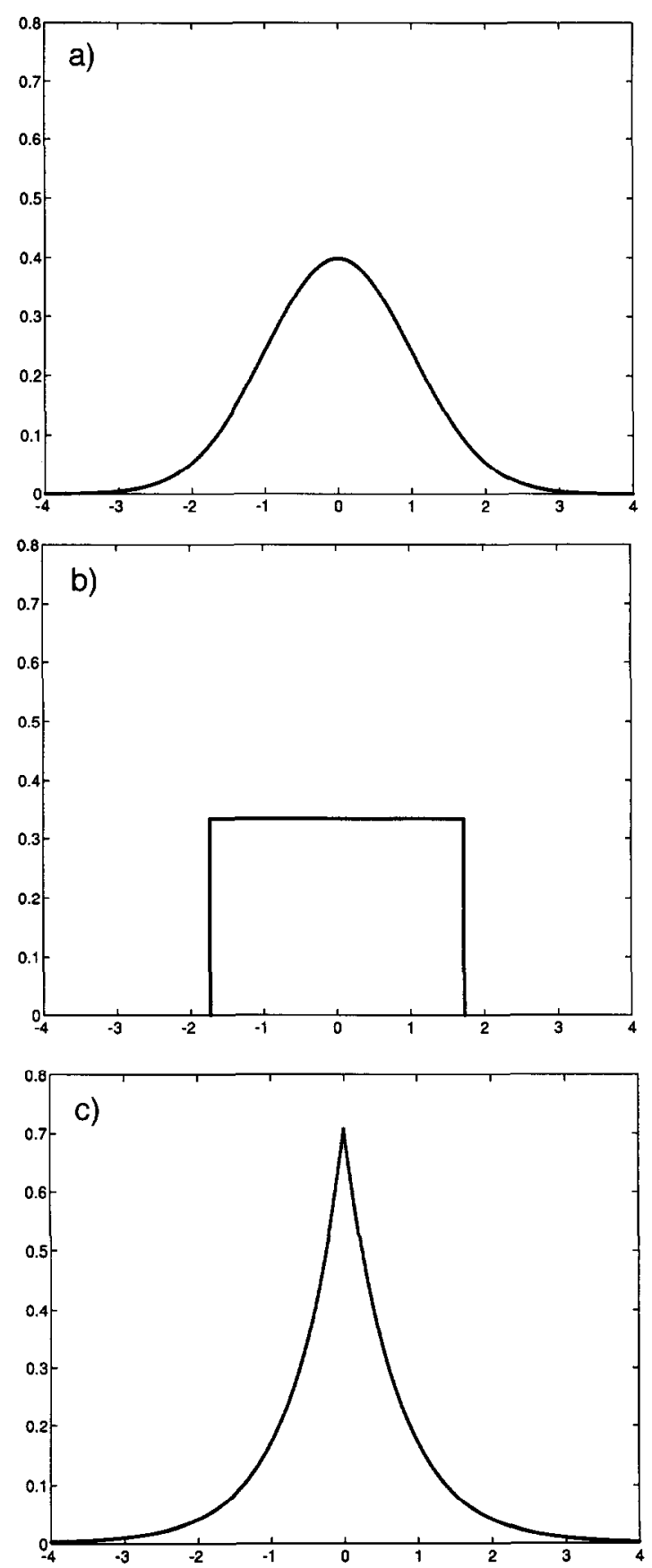

Fig. 6. Probability density function for: (a) normal distributed data, (b) uniform distributed data, (c) Laplace distributed data. 


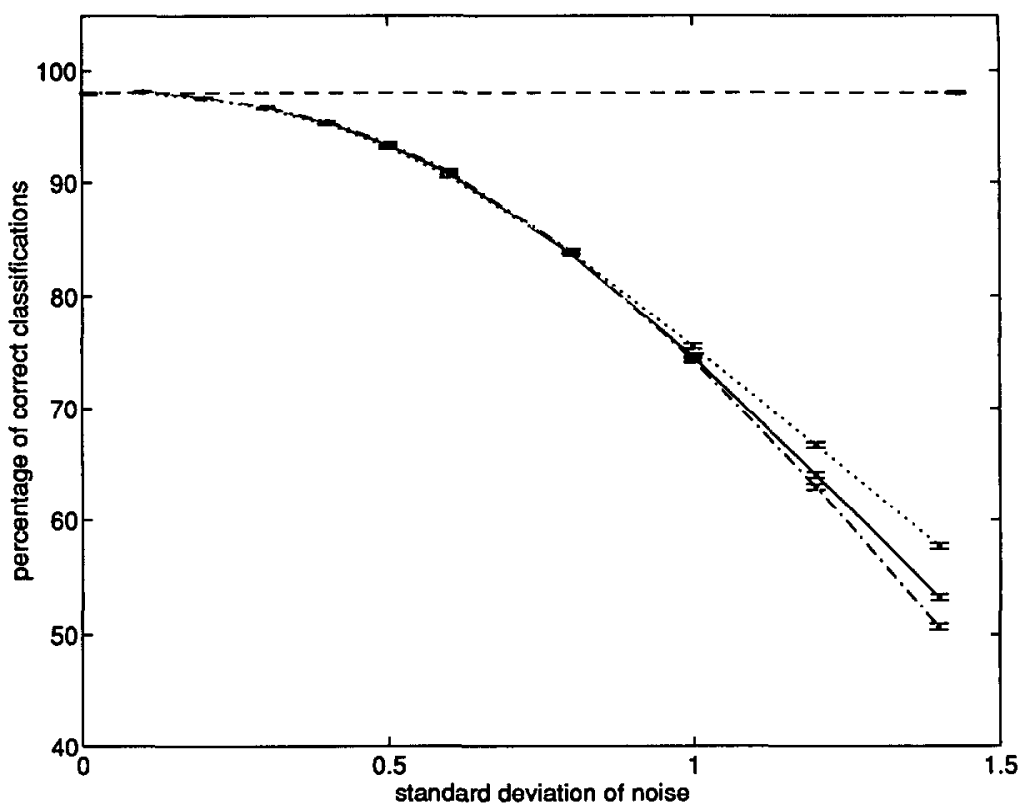

Fig. 7. Plot of the percentages of correct classifications together with their 99\%-confidence limits for RBF networks. Dashed line: value obtained with the test set (without noise). Dotted line: I aplace distribution of noise. Solid line: normal distribution of noise. Dashdot line: uniform distribution of noise.

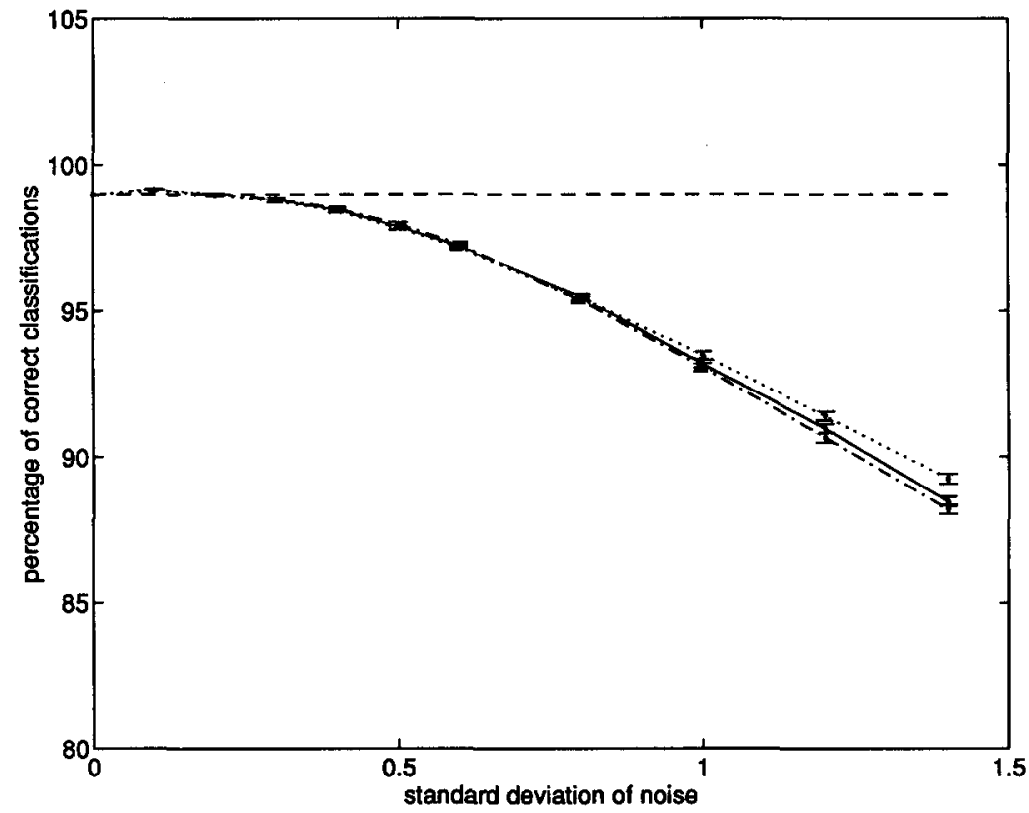

Fig. 8. Plot of the percentages of correct classifications together with their $99 \%$-confidence limits for MLF networks. Dashed line: value obtained with the test set (without noise). Dotted line: Laplace distribution of noise. Solid line: normal distribution of noise. Dashdot line: uniform distribution of noise. 
(ii) Noise duc to crrors without any privileged value, for instance, due to make a sum up to a round number. The corresponding probability density function is plotted in Fig. $6 \mathrm{~b}$.

(iii) Noise due to errors with one privileged value (the mean) but with still a rather high probability of obtaining values far away from the mean. Its probability density function is plotted in Fig. 6c.

In every case, the noise is sampled from the corresponding known distribution with a mean of zero and an increasing value of the standard deviation. This data set is added to the test set giving the noisy data set. The noisy data is presented to the trained network and the output of the network is calculated. With the output values, percentage of correct classifications, sensibility and specificity are calculated. This whole process is repeated for a thousand times for every kind of noise at different levels. This way an estimation of the mean and the confidence levels of the network output can be obtained (here, the confidence levels were calculated at $99 \%$ confidence).
This procedure can be written in a pseudo-code as: Initialization:

1. Choose a zero mean distribution function (normal, uniform or Laplace).

2. Fill an array with A values for the standard deviation of the input noise.

Simulations:

for standard_deviation_index $=1$ to A

for simulation $n u m b e r=1$ to number_of_simulations

1. Add noise, sampled with the chosen distribution, to the test set.

2. Present the noisy data to the trained network.

3. Compute and store the values of the percentage of correct classifications, sensibility and specificity.

End

Calculate the mean and standard deviation of the results.

End

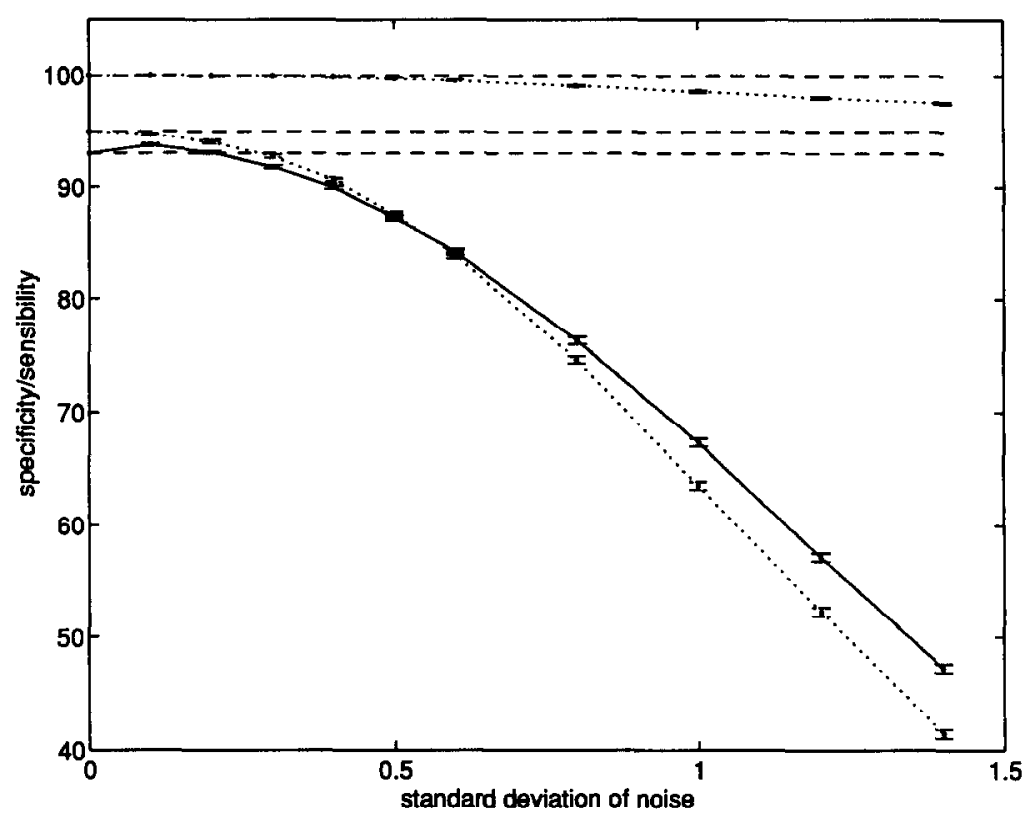

Fig. 9. Plot of the sensibility and specificity together with their $99 \%$-confidence limits computed with the euclidean distance $95 \%$ sample percentile criterion for both classes and for RBF networks. Dashed line: values obtained with the test set (without noise). Solid line: sensibility for class 2 . Dotted line: sensibility/specificity for class 1 . The upper dotted line corresponds to the specificity and the lower lines to the sensibility. Only the specificity for class 1 is shown because the specificity for class 2 coincides with it. 
Model the results obtained with their confidence limits as a function of the standard deviation of the input noise.

The behaviour of the percentages of correct classifications for RBF is shown in Fig. 7 for the three kinds of noise. It can be observed that as the standard deviation increases the values are dropping very fast, but in a similar trend for every kind of noise. For MLF networks (Fig. 8) the percentages drop also similarly for the different kinds of noise but much slower than for RBF, showing an almost linear tendency. Thus, related to the discriminating ability, it seems that MLF supports bigger amount of noise than that supported by RBF. But this is not necessarily a good property because for high values of the standard deviation, the input noisy data set has nothing to see with the original data set. For instance if an additional source of variability appears in the data, it can cause an increment of the errors which would go unnoticed for MLF while for RBF would not. This can be of great importance in quality control processes, for example.

In this sense, we can say that for small deviations in the input space, both RBF and MLF networks still produce acceptable results and act similarly. For higher deviations, RBF can recognize that the data set we are applying to is different from the data set the network was trained with, whereas MLF is still able to classify the objects.

For normal distribution of noise, Fig. 9 shows the sensibility and specificity computed with the euclidean distance $95 \%$ sample percentile criterion for both classes and for RBF networks. As can be observed, the specificity of the models remains almost on the values obtained with the original data set (without noise) whereas the sensibility gives acceptable values for small deviations and, again, drops fast when the deviations increase and in the same trend for both classes. The same behaviour is observed when one is computing the sensibility and specificity with the Mahalanobis distance criterion (Fig. 10).

Figs. 11 and 12 show the sensibility and specificity computed with the euclidean distance 95\% sample percentile and Mahalanobis distance 95\% sample percentile criteria respectively, for MLF networks and for both class 1 and class 2 . In this case,

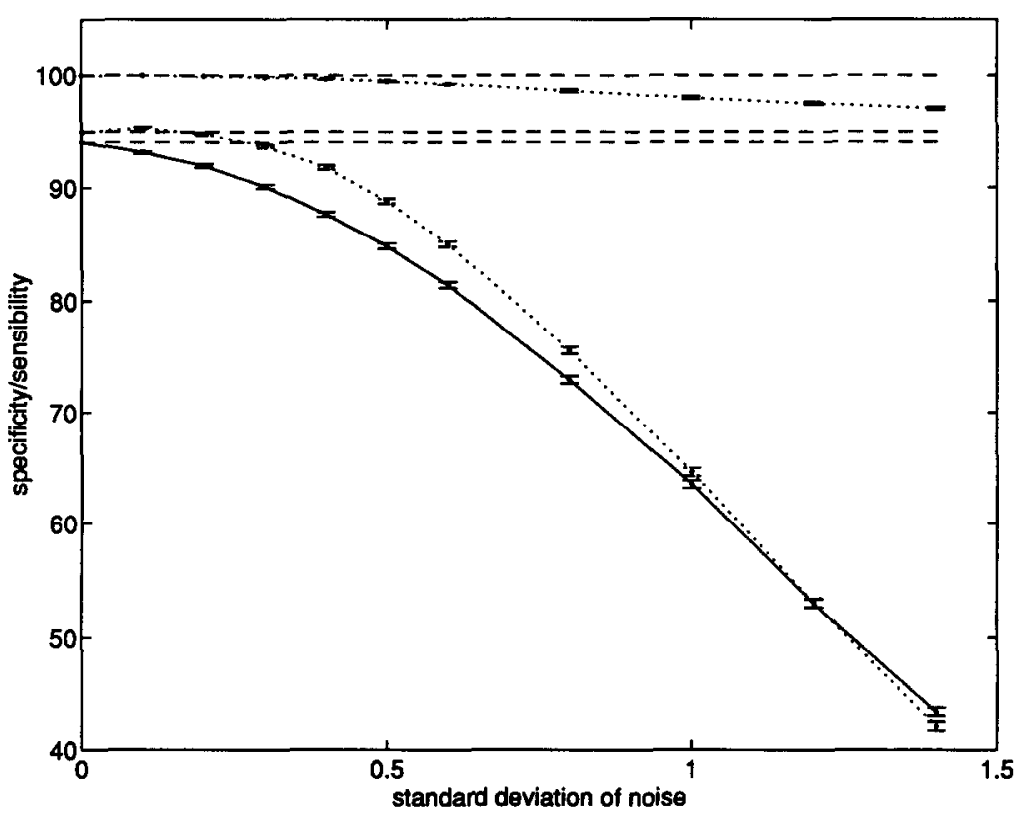

Fig. 10. Plot of the sensibility and specificity together with their $99 \%$-confidence limits computed with the Mahalanobis distance $95 \%$ sample percentile criterion for both classes and for RBF networks. Dashed line: values obtained with the test set (without noise). Solid line: sensibility for class 2 . Dotted line: sensibility/specificity for class 1 . The upper dotted line corresponds to the specificity and the lower lines to the sensibility. Only the specificity for class 1 is shown because the specificity for class 2 coincides with it. 


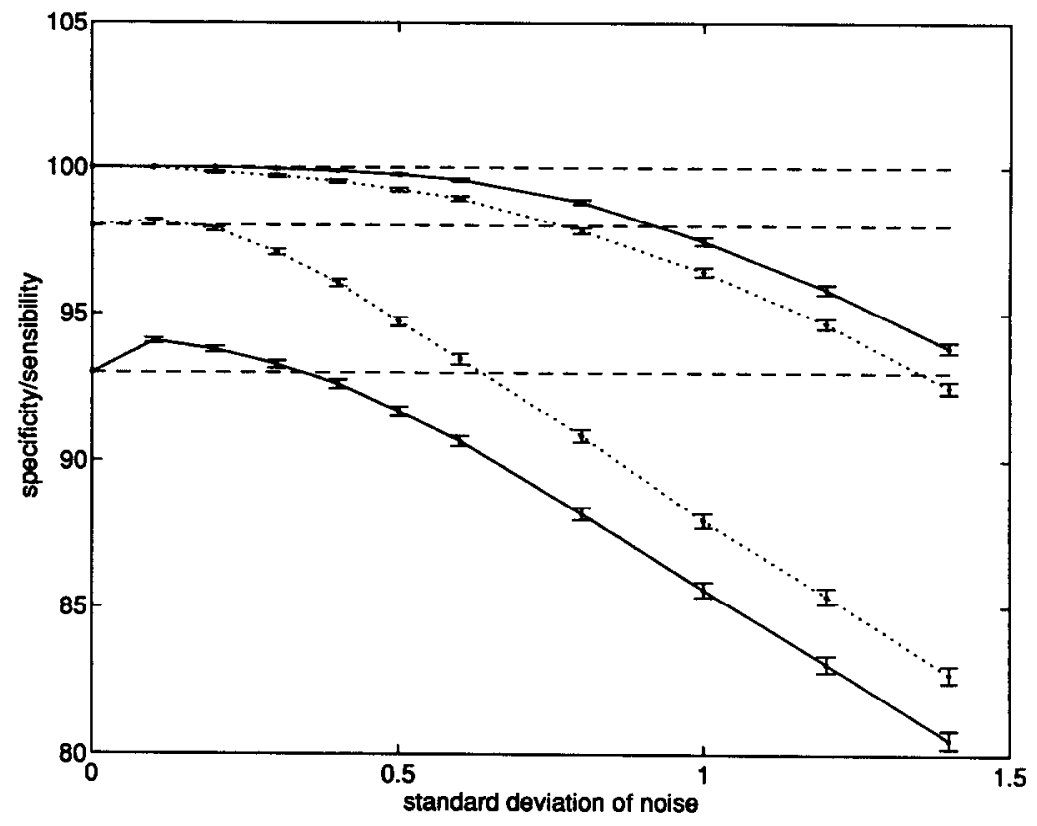

Fig. 11. Plot of the sensibility and specificity together with their $99 \%$-confidence limits computed with the euclidean distance $95 \%$ sample percentile criterion for both classes and for MLF networks. Dashed line: values obtained with the test set (without noise). The upper lines correspond to the specificity and the lower lines to the sensibility: solid line for class 2 and dotted line for class 1 .

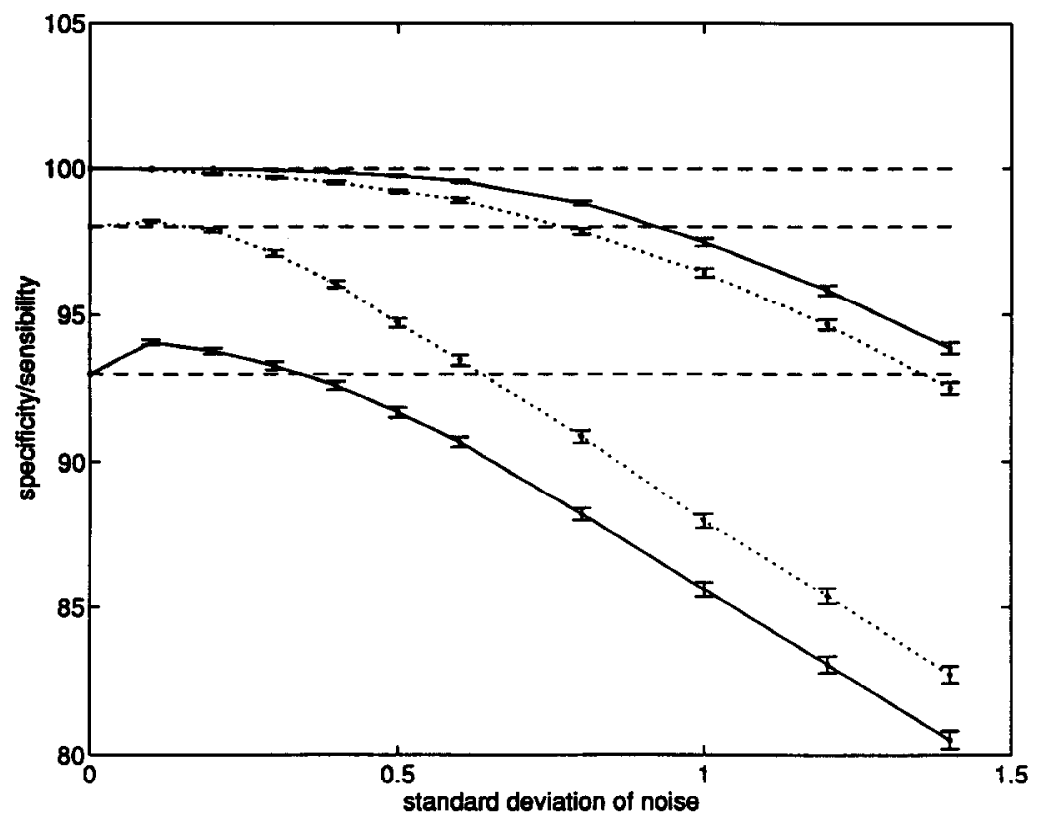

Fig. 12. Plot of the sensibility and specificity together with their $99 \%$-confidence limits computed with the Mahalanobis distance $95 \%$ sample percentile criterion for both classes and for MLF networks. Dashed line: values obtained with the test set (without noise). The upper lines correspond to the specificity and the lower lines to the sensibility: solid line for class 2 and dotted line for class 1 . 
the same tendency is observed in both graphs: sensibility and specificity of the classes drop more or less in the same range.

These different results for RBF and MLF networks can be explained by the way the networks operate: the outputs obtained with MLF networks are always in the line that join the theoretical centroids in the output space $((0,1)$ and $(1,0))$ so when the network starts getting worse, the outputs of the objects move themselves along the straight line and lie inside the class box of the other category. So, both the sensibility and specificity decrease. In RBF networks, if the objects are far away from the centroids of the radial base functions, they obtain a zero output and lie outside both models. Thus the sensibility is decreasing but the specificity remains $100 \%$.

The tendency of the results obtained with uniform and Laplace distributions were exactly the same as for normal distribution, so we do not show the figures to avoid including different figures with the same information.

Summarizing, both types of networks yield similar results when the various noise distributions are applied and related to both discriminating and modelling ability. MLF networks start dropping later than RBF networks but RBF can recognize that the objects are no in the same range that the objects in the training set.

\section{Software and hardware}

Principal component analysis has been performed with the PARVUS package [27]. All the programs for neural networks were written in MATLAB code and all the calculations were performed on IBM-compatible PCs 80486 at $50-66 \mathrm{MHz}$.

\section{Conclusions}

Ten MLF and ten RBF networks have been independently trained on a simulated data set with a priori known values for the sensibility and specificity. Estimating both the discriminating and modelling ability of the networks, the results show that the models are quite reproducible and, with the variability due to the estimators, the "a priori" established values for sensibility and specificity can be obtained. Also, PCA for independent ways of unfolding for RBF networks, show there are no structural differences among the ten networks trained to solve the same classification problem.

Furthermore, a robustness analysis has been carried out to study the sensitivity of this kind of networks to deviations in input space due to different distributional noise. In this case, MLF networks support a rather high amount of noise but RBF networks can detect faster that the input space is changing with respect to the data set used for training the networks.

Based on all the results it is hard to conclude which network is better for this classification task. In percentage of correct classifications MLF obtains higher values than RBF. The closure line between both types of networks is weak regarding to sensibility and specificity. And despite the fact that MLF networks seem to be more robust to noise in the input space, RBF networks are more sensitive to deviations in input space in the sense that they detect faster variations in the new object presented to the network. In this way, interpolation and extrapolation effects [28] can be detected. So when it comes to model validation, RBF can be considered more reliable.

Summarizing, the choice of a neural network concept (RBF or MLF) for classification tasks strongly depends on how the priorities about the model properties, with respect to sensibility, specificity and also parsimonity, are defined.

\section{References}

[1] E.P.P.A. Derks, M.S. Sánchez Pastor and L.M.C. Buydens, Chemom. Intelligent Lab. Sys., 28 (1995) 49-60.

[2] M.P. Derde, L. Kaufman and D.L. Massart, J. Chemom., 3 (1989) 375-395.

[3] M.P. Derde and D.L. Massart, Anal. Chim. Acta, 184 (1986) 33-51.

[4] M. Forina, S. Lanteri and L. Sarabia, J. Chemom., 9 (1995) $69-89$.

[5] A. Bos, Artificial Neural Networks as a Tool in Chemometrics, Ph.D. Thesis, University of Twente, Enschede, The Netherlands, 1993.

[6] D.A. White and D.A. Sofge, Handbook of Intelligent Control, Van Nostrand Reinhold, New York, 1992.

[7] J. Zupan and J. Gasteiger, Networks for Chemists. An Introduction, VCH, Weinheim, 1993.

[8] M. Carlin, T. Kavli and B. Lillekjendlie, Chemom. Intelligent Lab. Sys., 23 (1994) 163-177. 
[9] M. Hartnett, D. Diamond and P.G. Barker, Analyst, 118 (1993) 347-354.

[10] T.B. Blank and S.D. Brown, Anal. Chem., 65 (1993) 30813089.

[11] J. Park and I.W. Sandberg, Neural Comput., 5 (1993) 305316.

[12] K.M. Hornik, M. Stinchcombe and H. White, Neural Networks, 2 (1989) 359-366.

[13] H. Lohninger, J. Chem. Inf. Comput. Sci., 33 (1993) 736744.

[14] D.E. Rumelhart, G.E. Hinton and R.J. Williams, Learning internal representations by error propagation, in D.E. Rumelhart and J.L. McClelland (Eds.), Parallel Distributed Processing, Vol. 1, MIT Press, Cambridge, MA, 1986.

[15] N. Draper and H. Smith, Applied Regression Analysis, 2nd edn., New York, 1981.

[16] M.C. Ortiz, J.A. Sáez and J. López, Analyst, 118 (1993) 801-805.

[17] M.S. Sánchez and L.A. Sarabia, Chemom. Intelligent Lab. Sys., 28 (1995) 287-303.

[18] J.R.M. Smits, W.J. Melssen, L.M.C. Buydens and G. Kateman, Chemom. Intelligent Lab. Sys., 22 (1994) 165-189.
[19] L.-X. Sim et al., Chemom. Intelligent Lab. Sys., 25 (1994) $51-60$.

[20] T. Kohonen, Self-Organization and Associative Memory, Springer, Berlin, 1989.

[21] D.E. Goldberg, Genetic Algorithms in Search, Optimization and Machine Learning, Addison-Wesley, New York, 1989.

[22] H. Demuth and M. Beale, Neural Network Toolbox User's Guide, The MathWorks Inc., Natick, MA, 1993.

[23] M.I. Jordan and R.A. Jacobs, Neural Comput., 6 (1994)181214.

[24] R. Henrion, Chemom. Intelligent Lab. Sys., 25 (1994) 1-23.

[25] L.R. Tucker, Psychometrika, 31 (1966) 279-311.

[26] T.P. Hettmansperger, Statistical Inference Based on Ranks, Wiley, NY, 1984.

[27] M. Forina, R. Leardi, C. Armanino and S. Lanteri, PARVUS: An extendable Package of Programs for Data Exploration, Classification and Correlation, Release 1.2 available from the authors, Genoa, Italy, 1994.

[28] J.A. Leonard, M.A. Kramer and L.H. Ungar, Comput. Chem. Eng., 16 (1992) 819-836. 